\title{
The imprint of Gould's Belt on the local cosmic-ray electron spectrum
}

\author{
M. Pohl ${ }^{1, \star}$, C. Perrot ${ }^{2, \star \star}$, I. Grenier ${ }^{2}$, and S. Digel ${ }^{3}$ \\ ${ }^{1}$ Institut für Theoretische Physik, Lehrstuhl IV, Ruhr-Universität Bochum, 44780 Bochum, Germany \\ 2 Université de Paris VII \& CEA Saclay, Service d'Astrophysique, 91191 Gif-sur-Yvette, France \\ 3 W.W. Hansen Experimental Physics Laboratory, Stanford University, Stanford, CA 94305, USA
}

Received 15 May 2002 / Accepted 7 July 2003

\begin{abstract}
Recent analyses have shown that if the sources of cosmic-rays are discrete, as are supernova remnants, then the spectra of cosmic-ray electrons vary greatly with location and time and the locally measured electron spectrum may be not representative of the electron spectra elsewhere in the Galaxy. We have continued the earlier studies by investigating the impact of the star forming region Gould's Belt on the local electron spectrum at $\mathrm{GeV}$ to $\mathrm{TeV}$ energies. Our results indicate that if the electron sources in Gould's Belt are continous, the local electron spectrum would be slightly hardened with a spectral index increase of $\Delta s \approx 0.07$ due to the higher $\mathrm{SN}$ rate in the Belt. If the electron sources are discrete, which is the more probable case, the local electron spectrum above $\sim 30 \mathrm{GeV}$ is variable with similar amplitude as without Gould's Belt. By the method of Monte Carlo, we have also studied the correlation of the local electron flux with that in nearby molecular cloud complexes. The high energy electron flux above $30 \mathrm{GeV}$ near the Orion, Monoceros, Cassiopeia, Cepheus, and Perseus clouds does not vary in coordination with the local flux, but a correlation between the electron flux in the Taurus and Ophiuchus clouds and that in solar vicinity is indicated by our study, implying that the spatial correlation length is of the order of $200 \mathrm{pc}$ at electron energies of $100 \mathrm{GeV}$ and higher. This would permit hard $\gamma$-ray spectra from inverse Compton scattering to be produced in the more distant clouds, in agreement with the $\mathrm{GeV}$ excess observed towards Orion and Monoceros, without affecting the emissivity spectra of the very nearby clouds as in Taurus and Ophiuchus.
\end{abstract}

Key words. ISM: cosmic rays - Galaxy: general - gamma rays: theory

\section{Introduction}

The recent detections of non-thermal X-ray synchrotron radiation from the supernova remnants (SNR) SN1006 (Koyama et al. 1995), RX J1713.7-3946 (Koyama et al. 1997), IC443 (Keohane et al. 1997; Slane et al. 1999), Cas A (Allen et al. 1997), RCW86 (Borkowski et al. 2001), and others together with the subsequent detections of SN1006 (Tanimori et al. 1998) and RX J1713.7-3946 (Muraishi et al. 2000) at TeV energies support the hypothesis that at least Galactic cosmic-ray electrons are accelerated predominantly in SNR. To date there is no corresponding observational evidence for nucleon acceleration in SNR (Reimer \& Pohl 2002), though pion decay is the most probable explanation for $\mathrm{TeV}$-scale $\gamma$-ray emission from SNR such as Cas A (Aharonian et al. 2001), inside which the magnetic field strength is thought to be $0.1-1 \mathrm{mG}$ (Berezhko et al. 2003).

Send offprint requests to: $\mathrm{M}$. Pohl, e-mail: mkp@tp4.ruhr-uni-bochum.de

* Now at Department of Physics and Astronomy, Iowa State University, Ames, IA 50011-3160, USA.

$\star \star$ Now at W.W. Hansen Experimental Physics Laboratory, Stanford University, Stanford, CA 94305, USA.
SNR are transient phenomena, which happen stochastically in space and time. Therefore steady-state models of cosmicray electron propagation in the Galaxy may be inadequate and time-dependent transport calculation may be required. The latter show (Pohl \& Esposito 1998), that the spatial distribution of sources modifies the steady-state local electron spectrum, while the randomness in time induces a time variability in the local electron flux at higher energies that stems from the fluctuations in the number of SNR within a certain distance and time interval. Thus the discreteness of sources does not only cause a cutoff in the electron spectrum (Cowsik \& Lee 1979), but makes it variable with time and thus unpredictable beyond a certain energy.

While for steady-state models the observed electron spectrum requires an electron source spectral index of $s=2.4$ (Skibo 1993), in a time-dependent calculation the observed spectrum is inside the range of possible local spectra with source index $s=2.0$. This implies that the spatially averaged electron spectrum in the Galaxy, e.g. probed by line-of-sight integrals of leptonic emission through the Galactic plane, can be much harder than would be deduced in steady-state models. Pohl \& Esposito (1998) have argued that in this case the 
observed excess of gamma ray emission above $1 \mathrm{GeV}$ (Hunter et al. 1997) can in fact be explained as a correspondingly hard inverse Compton component. Based on a numerical solution of the transport equation Strong et al. (2000) find that the best fit of the spectrum of diffuse Galactic $\gamma$-rays is derived for an even harder electron injection spectral index $s=1.8$, for which, however, the locally measured electron spectrum can not be reproduced (Strong \& Moskalenko 2001). An electron source spectral index $s=2.0$ thus appears an appropriate choice. It would also correspond to the average radio synchrotron spectral index $\langle\alpha\rangle=0.5$ in shell-type SNR (Green 2001).

In the earlier studies the supernova rate was assumed constant in the Galactic plane. However, the Sun is located in a region of enhanced star formation activity, known as Gould's Belt, in which the supernova rate is three to five times larger than the local rate in the Galactic disc (Grenier 2000). As the fluctuations in the local high-energy electron spectrum arise from the small number of SNR actually contributing to it, one would be interested to know whether Gould's Belt can significantly damp the variations in or harden the electron spectrum. For that purpose we have calculated the following using a kinematic model for the geometry and expansion of Gould's Belt (Grenier \& Perrot 2001; Perrot \& Grenier 2003):

- the electron spectrum in the solar vicinity both for the steady-state and for the time-dependent case (Sect. 2);

- the electron spectra near seven local molecular cloud complexes, namely Orion, Monoceros, Taurus, Perseus, Cepheus, Cassiopeia, and Ophiuchus. In particular, we have calculated the correlation of the electron spectra at those clouds with that in the solar vicinity. Studies of the highenergy $\gamma$-ray emission observed toward these molecular clouds have recently been published. The presence or absence of a GeV excess in these various clouds is compared with the results of the correlation study (Sect. 3).

\section{The local cosmic-ray electron spectrum}

\subsection{The propagation model}

Because effects of the spatial and temporal structure of the electron sources appear only at higher particle energies, at which the radiative loss time is short, we may describe the propagation of electrons at energies higher than a few $\mathrm{GeV}$ by a simplified, time-dependent transport equation

$$
\frac{\partial N}{\partial t}-\frac{\partial}{\partial E}\left(b E^{2} N\right)-D E^{a} \nabla^{2} N=Q
$$

where we consider continous energy losses by synchrotron radiation and inverse Compton scattering, a diffusion coefficient $D E^{a}$ dependent on energy, and a source term $Q$. The Green's function for this problem can be found in the literature (Ginzburg \& Syrovatskii 1964).

$G=\frac{\delta\left(t-t^{\prime}+\frac{E-E^{\prime}}{b E E^{\prime}}\right)}{b E^{2}(4 \pi \lambda)^{3 / 2}} \exp \left(-\frac{\left(r-r^{\prime}\right)^{2}}{4 \lambda}\right)$

with

$\lambda=\frac{D\left(E^{a-1}-E^{a-1}\right)}{b(1-a)}$.
In the case of discrete sources the injection term $Q$ is a sum over all sources. For an individual source commencing operations at time $t_{0}$ and injecting for a time period $\tau$ we can write

$Q_{i}=q_{0} E^{\prime-s} \delta\left(r^{\prime}\right) \Theta\left(t^{\prime}-t_{0}\right) \Theta\left(t_{0}+\tau-t^{\prime}\right)$.

Without loss of generality we can set $t=0$ and obtain

$N=q_{0} E^{-s}$

$\times \int_{-\frac{1}{b E}}^{0} \mathrm{~d} t^{\prime} \frac{\Theta\left(t^{\prime}-t_{0}\right) \Theta\left(t_{0}+\tau-t^{\prime}\right)}{(4 \pi \Lambda)^{3 / 2}\left(1+b E t^{\prime}\right)^{2-s}} \exp \left(-\frac{r^{2}}{4 \Lambda}\right)$

where

$\Lambda=\frac{D E^{a-1}}{b(1-a)}\left(1-\left(1+b E t^{\prime}\right)^{1-a}\right)$

and $r$ is the distance between source and observer. $N$ is the contribution to the electron spectrum provided by a single source (SNR) at distance $r$ which is (was) injecting electrons for a time period $\tau$ starting at $t_{0}$. The total spectrum can then be obtained by summing the contributions from all individual sources.

For sources in the Galactic disc, the location and explosion time was assumed random with a uniform space distribution inside a disc with radius $r_{\mathrm{s}}=15 \mathrm{kpc}$ and half-thickness $z_{\mathrm{s}}=80 \mathrm{pc}$. The sun is located $16 \mathrm{pc}$ above the Galactic plane (Hammersley et al. 1995; Freudenreich 1998). The distribution of sources in Gould's Belt is described below.

For each volume element $\delta V$ the expected number of supernovae within the (short) time interval $\delta t$ is

$N_{\lambda}=\eta \delta V \delta t$

where $\eta$ is the supernova rate per volume element, which in the case of the homogeneous disc distribution is

$\eta=\frac{1}{V_{\text {tot }} t_{\text {inj }}}$

where $V_{\text {tot }}$ is the total volume of the source distribution and $t_{\mathrm{inj}}=55$ years is the inverse of the Galactic supernova rate (Cappelaro et al. 1997). For Gould's Belt, $\eta$ itself is a timedependent quantity, determined by the enhancement of the total supernova rate in the Belt and the expansion of the structure. At a given time the number of supernovae in the volume element $\delta V$ is a Poissonian random number with mean $N_{\lambda}$. The final electron spectrum is then derived by summing over the contributions of the individual SNR per volume element and summing over all relevant volume elements.

In a typical simulation 1000 "random" spectra are calculated, from which the distribution of possible spectra and, by simple averaging of the time-dependent solutions, the timeaveraged spectrum are derived. The effect of solar modulation is taken into account for all simulated spectra using a forcefield parameter $\Phi=400 \mathrm{MeV}$ (Gleeson \& Axford 1968).

The propagation parameters used in the simulations are mostly the same as in Pohl \& Esposito (1998). The total energy density of the ambient photon fields plus that of the perpendicular component of the magnetic field strength is taken to be $3.5 \mathrm{eV} / \mathrm{cm}^{3}$. The changes in the Compton cross section in 


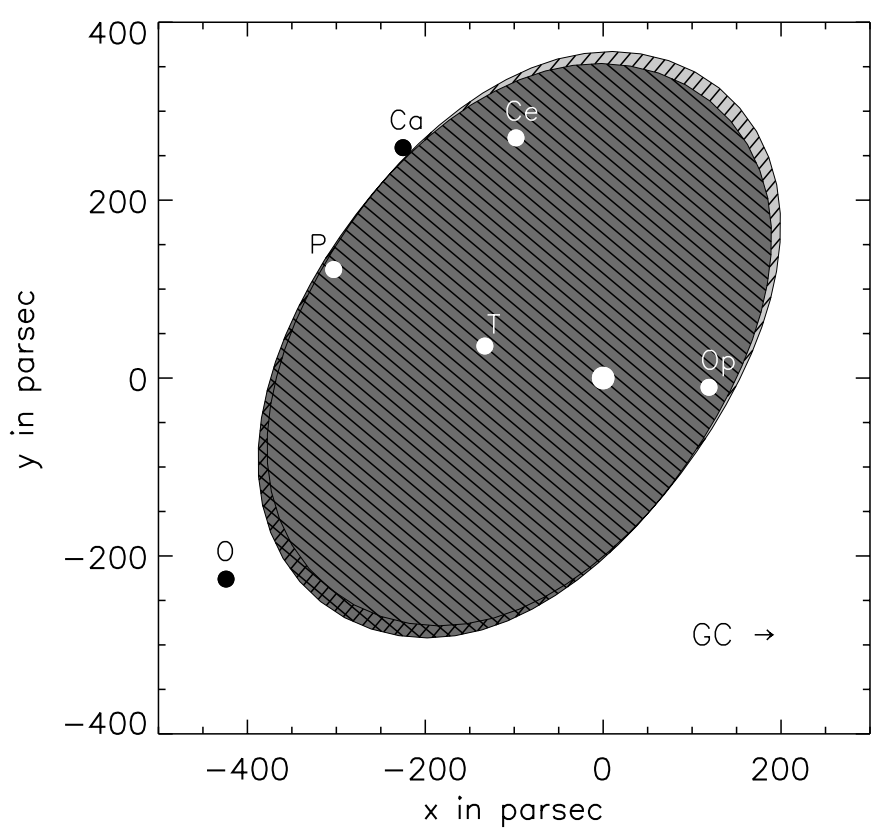

Fig. 1. The present extent of Gould's Belt shown as projection onto the Galactic plane. The position of the sun is indicated by the big filled circle.The coordinates are righthanded heliocentric with $\mathrm{x}$ pointing toward the Galactic Center. The small circles indicate the positions of the molecular complexes (O: Orion, P: Perseus, Ca: Cassiopeia, T: Taurus, Ce: Cepheus, Op: Ophiuchus). The Monoceros complex lies beyond Orion.

the Klein-Nishina regime of optical and near-infrared photon fields are neglected.

Our calculations depend somewhat on the choice of diffusion coefficient. At high energies, electrons and nuclei will scatter off the same turbulence except for the helicity, and thus their diffusion behaviour is expected to be similar. In Pohl \& Esposito (1998) the spatial diffusion coefficient was $D=4 \times 10^{27} \mathrm{~cm}^{2} \mathrm{~s}^{-1}$ at $1 \mathrm{GeV}$ and assumed to increase with energy to the power $a=0.6$, in accord with results for a $2 \mathrm{D}$ diffusion model fit to the local spectra of 13 primary and secondary cosmic ray nuclei at rigidities between $1 \mathrm{GeV}$ and $10^{3} \mathrm{GeV}$ (Webber et al. 1992). In the past few years a number of new studies of cosmic-ray propagation have been published, which suggest that the diffusion coefficient has a higher value than previously thought. Since we are mostly interested in the highenergy regime, it will be useful to compare the results of different studies for the diffusion coefficient at $300 \mathrm{GeV}$, which allows us to include reacceleration models, for the reacceleration is only important around a $\mathrm{GeV}$ and below. Typical results (in units of $10^{29} \mathrm{~cm}^{2} \mathrm{~s}^{-1}$ ) in the most recent literature are: $D(300 \mathrm{GeV})=2.6$ for a $4 \mathrm{kpc}$ halo in a reacceleration model (Maurin et al. 2001), $D(300 \mathrm{GeV})=1.8$ for a disk-halo model and $D(300 \mathrm{GeV})=3.3$ with stochastic reacceleration (Jones et al. 2001), $D(300 \mathrm{GeV})=3.2$ for a $3 \mathrm{kpc}$ halo in a diffusionconvection model and $D(300 \mathrm{GeV})=2.1$ in a reacceleration model (Strong \& Moskalenko 1998). In this paper we will use the average of these results, which is $D(300 \mathrm{GeV})=2.6$, corresponding to $D(1 \mathrm{GeV})=8.5 \times 10^{27} \mathrm{~cm}^{2} \mathrm{~s}^{-1}$, i.e. a factor of two larger than in the study of Pohl \& Esposito (1998).

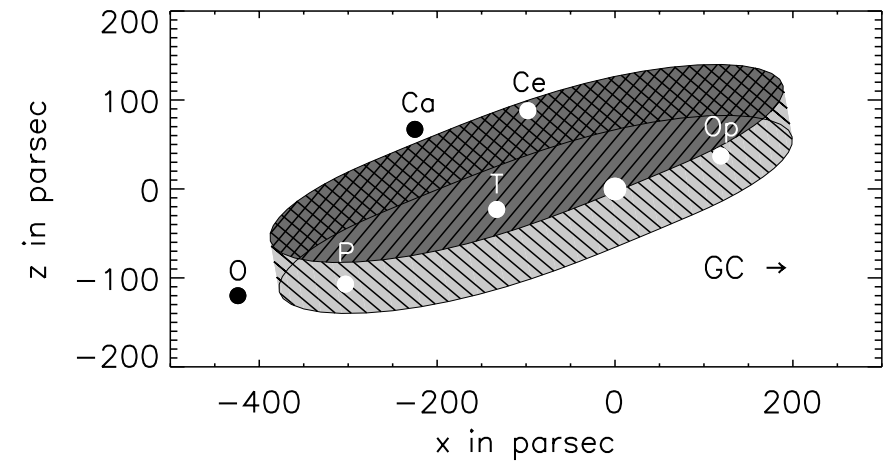

Fig. 2. The present day state of the Belt model projected onto the $x z-$ plane. The coordinate system and the labels are as in Fig. 1. The $z$-axis points to the North Galactic Pole.

For a $3 \mathrm{kpc}$ halo this choice of diffusion coefficient implies that electrons with energies higher than $2 \mathrm{GeV}$ loose their energy before they reach the escape boundary. We are interested in electrons of substantially higher energy, and thus the neglect of an escape term in Eq. (1) is justified.

The injection spectral index of electrons is $s=2.0$, which corresponds to the mean synchrotron spectral index of individual SNR (Green 2001). The electron injection rate, $Q$, is adjusted to match the observed local electron flux at $15 \mathrm{GeV}$. All SNR are assumed to provide the same electron injection rate over the same period of time, namely $\tau=5 \times 10^{4}$ years. The evolution of any real SNR may be extremely complex, particularly because of the varying properties of the ambient medium. Generally, the acceleration efficiency will decrease with time, but the accelerated particles can only slowly escape to the upstream region, i.e. to the interstellar medium. A typical SNR will therefore inject particles for a substantially longer time than indicated by the deceleration time scale of its forward shock. Our choice for the SNR lifetime, $\tau$, is a somewhat conservative estimate, because a smaller lifetime would lead to larger fluctuations in the cosmic-ray electron spectra.

\subsection{The effect of Gould's Belt}

The most prominent local system of star-forming regions is Gould's Belt (Pöppel 1997), an expanding disc-like region tilted at about $18^{\circ}$ to the Galactic plane and about $600 \mathrm{pc}$ in diameter, in which the supernova rate per area is three to five times higher than the Galactic rate at the solar circle (Grenier 2000). Based on its stellar content and on its expansion characteristics the age of this structure can be estimated to be 30 to 40 Myr. Figures 1 and 2 indicate the geometry of Gould's Belt at the present day in heliocentric coordinates.

The kinematical expansion of Gould's Belt can be modelled assuming an initial explosive event (Grenier \& Perrot 2001; Perrot \& Grenier 2003). The nature of that explosive event is unclear. Most likely in view of the inclination of Gould's Belt is the impact of a massive high-velocity cloud on the Galactic plane (Comerón \& Torra 1992; Comerón 1999). The dynamical model was fitted to the velocities of the local $\mathrm{H} \mathrm{I}$ and $\mathrm{CO}$ clouds that participate in the expansion. When available, an estimate of the distance to the clouds was used, based on Hipparcos 


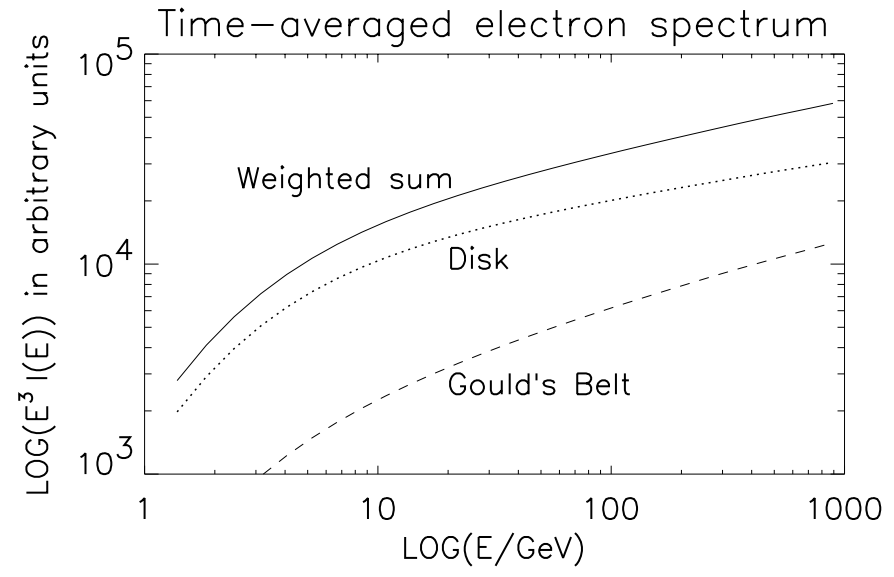

Fig. 3. The time-averaged local electron spectrum for a homogeneous Galactic disc distribution of SNR (dotted line) and for a Gould's Belt distribution of SNR (dashed curve), both with the same SN rate per area. The solid line shows the weighted sum of both, the weights corresponding to the supernova rate being a factor of 3.2 higher in the area covered by Gould's Belt than in the local Galactic disc. The effect of Gould's Belt is a spectral index increase $\Delta s \approx 0.07$ between $10 \mathrm{GeV}$ and $\mathrm{a} \mathrm{TeV}$.

measurements. This model describes the lateral expansion of an inclined, cylindrical shock wave that sweeps momentum from the interstellar medium. Because of the Galactic differential rotation, the initially circular section of the Belt becomes rapidly elliptical. Additionally, a warp of the Belt is observed as a consequence of the pull exerted by the Galactic gravitational potential and of the ambient interstellar density gradients. The thickness of the Belt was fixed at $60 \mathrm{pc}$ since it best fits the data.

The age of the Belt is considerably higher than the time window of interest in this analysis, namely the radiative energy loss time of electrons with energies higher than $10 \mathrm{GeV}$. Given the slow expansion of Gould's Belt over the last ten million years, the details of the initial geometrical evolution are not important for the present analysis. The important parameters are the inclination and the elliptical size of the Belt, and to a lesser extent the warp and the age of the Belt. Those parameters have been found in agreement with other estimates (Olano 1982; Moreno et al. 1999) and with the positions of massive stars in the Belt (de Zeeuw et al. 1999). Test simulations with modified models of Gould's Belt indicate that the results reported here are indeed insensitive to the particulars of the expansion model, as long as the present extent of the Belt is as shown in Figs. 1 and 2 and the coasting velocity of the rim fits the observed velocities of the local $\mathrm{HI}$ and $\mathrm{CO}$ clouds that participate in the expansion.

We have assumed that enhanced SN activity commences without delay in the regions which are over-run by the expanding front of Gould's Belt, hence the Belt is treated as a thick disk as opposed to a quasi-circular shell. So, supernovae are assumed to randomly occur with a uniform space distribution inside the expanding Belt. In reality this may not be true, in which case the effect of Gould's Belt would be less than calculated here. The supernova rate in the local Galactic plane is assumed to be $25 \mathrm{kpc}^{-2} \mathrm{Myr}^{-1}$, as in Pohl \& Esposito (1998), whereas it is taken as $80 \mathrm{kpc}^{-2} \mathrm{Myr}^{-1}$ in the area covered by the Belt (Grenier 2000), so that the supernova rate per volume, $\eta$, is the sum of that for a homogeneous disc (Eq. (8)) and that for Gould's Belt.

In Fig. 3 we show the results for the time-averaged (steadystate) local electron spectrum, which is obtained by averaging the 1000 simulated spectra for the time-dependent case. At higher energies the radiative energy losses permit only nearby SNRs to contribute to the local electron flux. Thus a locally enhanced SNR rate is increasingly important with increasing energy. Consequently, the contribution of SNR in Gould's Belt yields a slightly harder spectrum than that of the Galactic SNR alone. The hard spectrum of electrons from Gould's Belt SNR is entirely a proximity effect.

In Fig. 4 we compare the range of possible local electron spectra for a homogeneous Galactic disc distribution of SNR with that with the addition of Gould's Belt. The flux variability amplitudes are very similar with and without the inclusion of Gould's Belt. That would be different, had we chosen a smaller diffusion coefficient, for in that case the increase in the number of local sources would lead to a smaller variability amplitude.

The electron source power per SNR is nearly the same as that needed for Galactic disc supernovae only. Gould's Belt reduces the power requirement to $\sim 65 \%$, but the higher diffusion coefficient used in this paper mandates an increase of the SNR source power to $\sim 140 \%$, so that the balance of the two is a reduction to $\sim 90 \%$ of the SNR power needed in the original paper (Pohl \& Esposito 1998). The power was $P \simeq 2 \times 10^{36} \mathrm{erg} / \mathrm{s}$ for 50000 years of SNR lifetime or $W \simeq 3 \times 10^{48} \mathrm{erg}$ in terms of total energy. In both cases, the locally observed electron spectrum is in the range of possible spectra, though with Gould's Belt the probability is lower, because the calculated spectra are on average slightly harder.

\section{The electron spectrum near local molecular cloud complexes}

We can calculate the time-averaged electron spectra at local molecular cloud complexes and, in the time-dependent case, the correlation of these spectra with that observed locally. Again we use a random number generator to stochastically place supernovae in space and time following the given probability distribution, here the Galactic disc plus Gould's Belt. In each run, we calculate the electron spectrum at various locations using the same set of supernovae, and thus derive the local electron spectrum, $F_{\odot}(E)$, as well as those near the molecular clouds, $F_{\mathrm{mc}}(E)$, where mc indicates the molecular cloud complex in question. After $N=1000$ runs we can calculate the relative deviation of the time-averaged spectrum, $\left\langle F_{\mathrm{mc}}\right\rangle$, near those clouds (mc) from the time-averaged local spectrum, $<F_{\odot}>$, as

$\Delta_{\mathrm{mc}}=\left(\frac{<F_{\mathrm{mc}}>}{<F_{\odot}>}\right)$. 


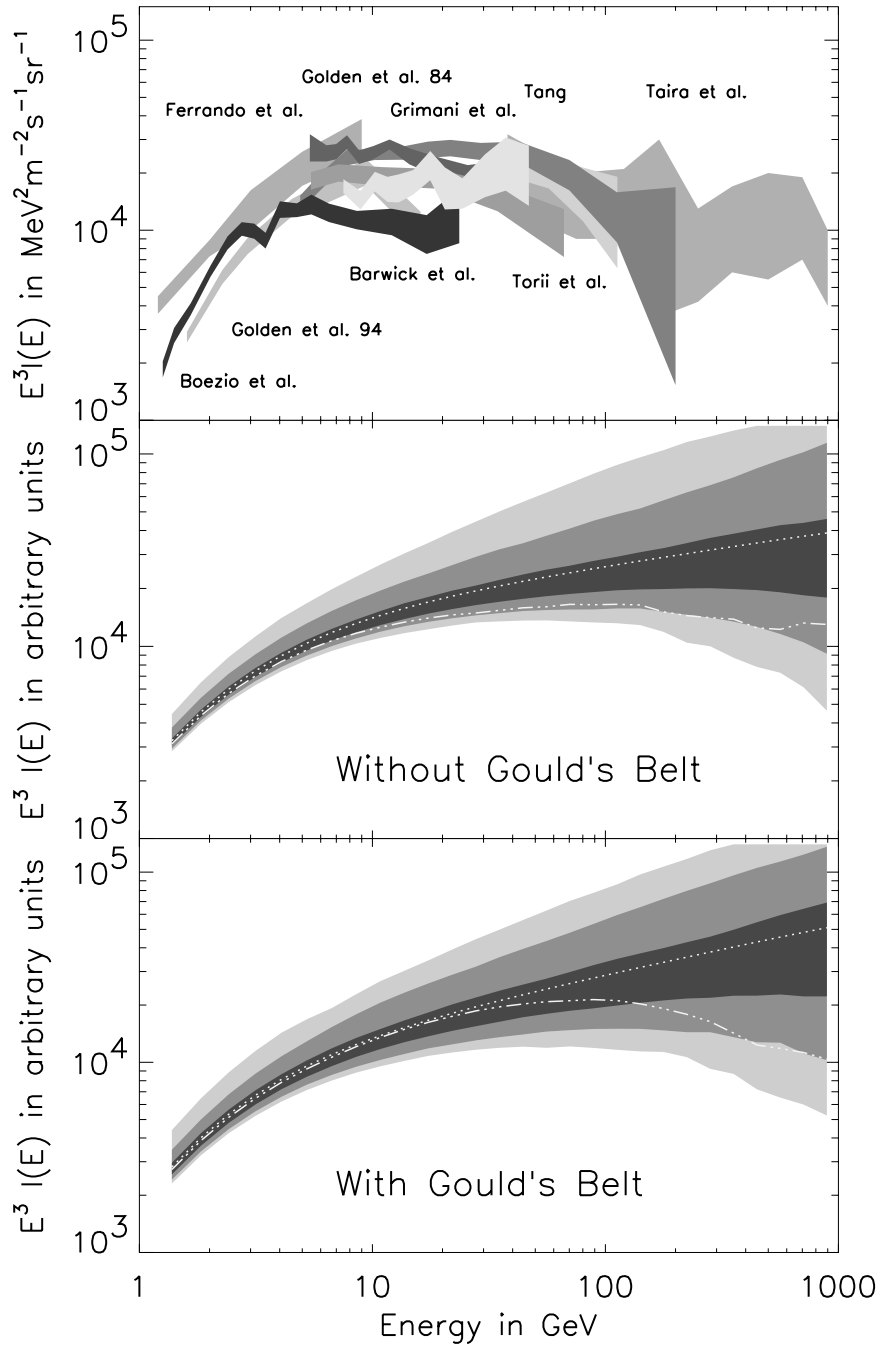

Fig. 4. The observed local electron spectrum (top) compared with the range of possible spectra for a homogeneous Galactic disc distribution of SNR with an initial spectral index of $s=2.0$ at the sources (middle) and with the range obtained for a Galactic disc distribution plus Gould's Belt (bottom). At each energy, the locally observed spectra will be in the dark-grey shaded region during $50 \%$ of the time, in the medium-grey shaded area during $90 \%$ of the time, and during $99 \%$ of the time within the light-grey shaded region. The white dash-dotted lines show one of 1000 random spectra as a particular example of what may be observed. The white dotted lines indicate the time-averaged spectrum.

We can also calculate the absolute dispersion in the local electron flux

$\sigma_{\mathrm{abs}}=\sqrt{\frac{1}{N} \sum_{n=1}^{N}\left(\frac{F_{\odot}(n)-<F_{\odot}>}{<F_{\odot}>}\right)^{2}}$.

It can be compared with

$\sigma_{\mathrm{mc}}=\sqrt{\frac{1}{N} \sum_{n=1}^{N}\left(\frac{F_{\mathrm{mc}}(n)-<F_{\mathrm{mc}}>}{<F_{\mathrm{mc}}>}-\frac{F_{\odot}(n)-<F_{\odot}>}{<F_{\odot}>}\right)^{2}}$
Table 1. The seven molecular cloud complexes and their representative positions.

\begin{tabular}{l|ccc|c}
\hline \hline Molecular complex & Longitude & Latitude & Distance & Ref. \\
\hline Perseus $(\mathbf{P})$ & $158^{\circ}$ & $-21^{\circ}$ & $350 \mathrm{pc}$ & $\mathrm{a}$ \\
Taurus $(\mathbf{T})$ & $165^{\circ}$ & $-9.5^{\circ}$ & $140 \mathrm{pc}$ & $\mathrm{a}$ \\
Orion $(\mathbf{O})$ & $208^{\circ}$ & $-16^{\circ}$ & $500 \mathrm{pc}$ & $\mathrm{b}$ \\
Monoceros (M) & $215^{\circ}$ & $-12^{\circ}$ & $830 \mathrm{pc}$ & $\mathrm{b}$ \\
Cassiopeia $(\mathbf{C a})$ & $131^{\circ}$ & $11^{\circ}$ & $350 \mathrm{pc}$ & $\mathrm{c}$ \\
Cepheus $(\mathbf{C e})$ & $110^{\circ}$ & $17^{\circ}$ & $300 \mathrm{pc}$ & $\mathrm{c}$ \\
Ophiuchus $(\mathbf{O p})$ & $355^{\circ}$ & $17^{\circ}$ & $125 \mathrm{pc}$ & $\mathrm{d}$ \\
\hline
\end{tabular}

a Ungerechts \& Thaddeus (1987).

b Maddalena et al. (1986).

c Grenier et al. (1989).

d de Geus et al. (1990).

which would indicate the difference of the relative variation of the electron spectrum at the cloud and in the solar vicinity. We know that for two fluctuating quantities $\delta_{1,2}$

$<\left(\delta_{1}-\delta_{2}\right)^{2}>=<\delta_{1}^{2}>+<\delta_{2}^{2}>-<2 \delta_{1} \delta_{2}>$

where the last term on the right hand side describes the actual correlation. Applied to Eq. (11) we see that if the electron spectrum $F_{\mathrm{mc}}(E)$ varies independently of the local one, $F_{\odot}$, (i.e. $<\delta_{1} \delta_{2}>=0$ ), $\sigma_{\mathrm{mc}}$ would be somewhat larger than $\sigma_{\text {abs }}$, depending on the dispersion in the spectrum at the molecular cloud. If, on the other hand, the spectrum $F_{\mathrm{mc}}$ varies in coordination with the local one, $F_{\odot}, \sigma_{\mathrm{mc}}$ would be smaller than $\sigma_{\text {abs }}$, for the absolute dispersions in the spectra at the clouds and in the solar vicinity are similar.

We calculate the electron spectrum in each cloud complex only for one representative position, which is determined by the peak in column density of molecular gas, for the actual calculation of sufficiently many spectra is rather time-consuming. The coordinates of the representative positions of all seven cloud complexes are given in Table 1.

Figure 5 shows the relative deviation of the time-averaged spectra near the seven clouds from the local spectrum. For all clouds but one the average spectrum is softer than that in the solar vicinity, which is caused by two effects: first, the more central to the Gould's Belt the cloud is located, the higher is the contribution of the electrons from Gould's Belt itself, which, as shown in Fig. 3, has on average a harder spectrum than the contribution of the disk. Second, the larger the distance of the cloud from the Galactic midplane is, the softer are the spectra. That is, because very close-by electron sources are underrepresented. For Orion and in particular for Monoceros both effects add up to make their spectra softer on average, whereas Taurus is located at a favorable position for both effects, and the average spectrum there is correspondingly harder than that in the solar vicinity. However, the differences in the average electron spectrum are small compared with the temporal and spatial fluctuations of the spectra (Fig. 4), the spectral index varying at a level of $\Delta s \lesssim 0.08$ between a few $\mathrm{GeV}$ and one TeV.

Figure 6 shows the amplitude of differences in dispersions, $\sigma$, in the electron spectra at the reference positions of the clouds and in the solar vicinity. Most interesting are the results for 


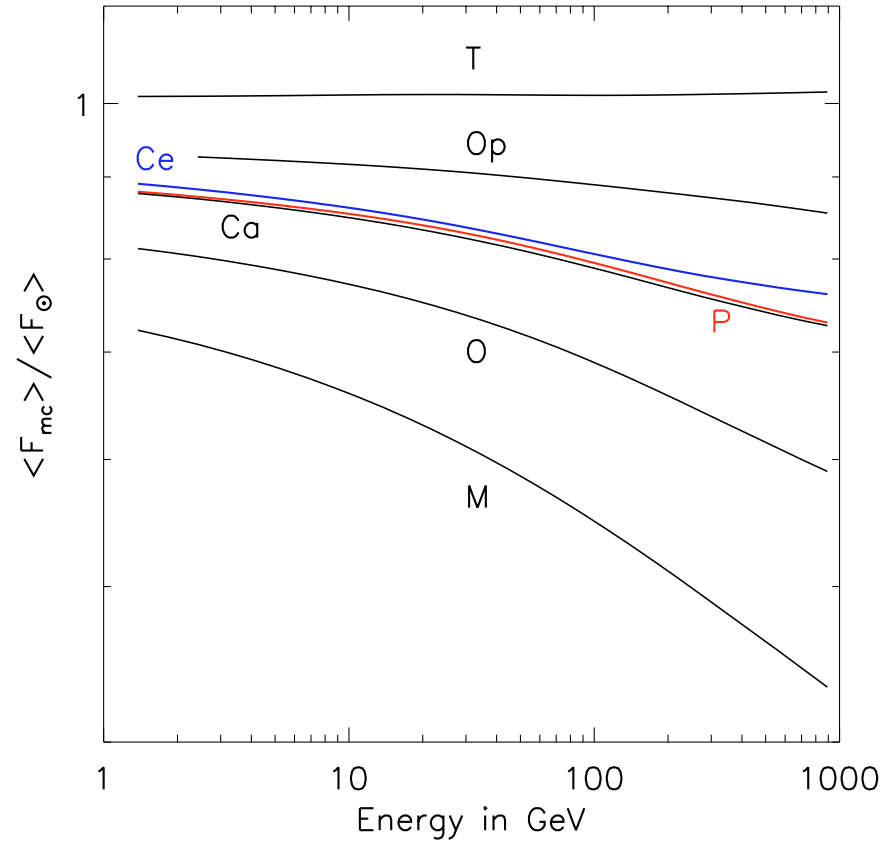

Fig. 5. The ratio of the time-averaged electron spectrum at the reference positions for the seven clouds to the local average electron spectrum. The reference positions for Orion and Monoceros are located above the thin disk distribution of Galactic supernovae, as a consequence of which the average spectra are softer and the flux is less than in the solar vicinity at all energies.

electron energies higher than $10 \mathrm{GeV}$ because at lower energies the variability is caused by the rare supernova explosions in the immediate vicinity of the reference locations. For Monoceros, Orion, Cassiopeia, Cepheus, and Perseus $\sigma_{\mathrm{mc}}$ is larger than $\sigma_{\text {abs }}$, indicating that the spectra near those clouds are not correlated with that measured locally. For Taurus $\sigma_{\text {Tau }}$ is slightly less than $\sigma_{\text {abs }}$ for energies higher than $50 \mathrm{GeV}$, and for Ophiuchus $\sigma_{\mathrm{Oph}}$ is significantly less than $\sigma_{\mathrm{abs}}$, indicating that the spectra at the two clouds vary in coordination with that in the solar vicinity. Thus at the nearby clouds $(D \simeq 140 \mathrm{pc}$ and $D \simeq 125 \mathrm{ps}$, resp.) we find the electron spectra correlated with that in the solar vicinity, whereas at the other, more distant clouds $(D \gtrsim 300 \mathrm{pc}$ ) we see no evidence of a correlation. The correlation length for the variations in the electron spectrum must therefore be $l_{\mathrm{c}} \approx 200 \mathrm{pc}$ at energies around $100 \mathrm{GeV}$ and higher. This result is independent of a possible variation or dispersion in the injection spectral indices of electrons.

Having shown that the cosmic-ray electron spectra near the five molecular clouds with distances $D \gtrsim 200 \mathrm{pc}$ and that in the solar vicinity are uncorrelated, we can conclude that inverse Compton scattering of locally emitted infrared radiation near those molecular clouds can yield hard $\gamma$-ray spectra, even if the electron spectrum in the solar vicinity is rather soft. On the other hand, the electron spectra at the Ophiuchus and Taurus complexes should be similar to that measured locally, hence no $\mathrm{GeV}$ excess should be observable toward these clouds, if it is caused by inverse Compton scattering.

The particular molecular cloud complexes selected here are nearby and fairly isolated at medium Galactic latitudes. Using

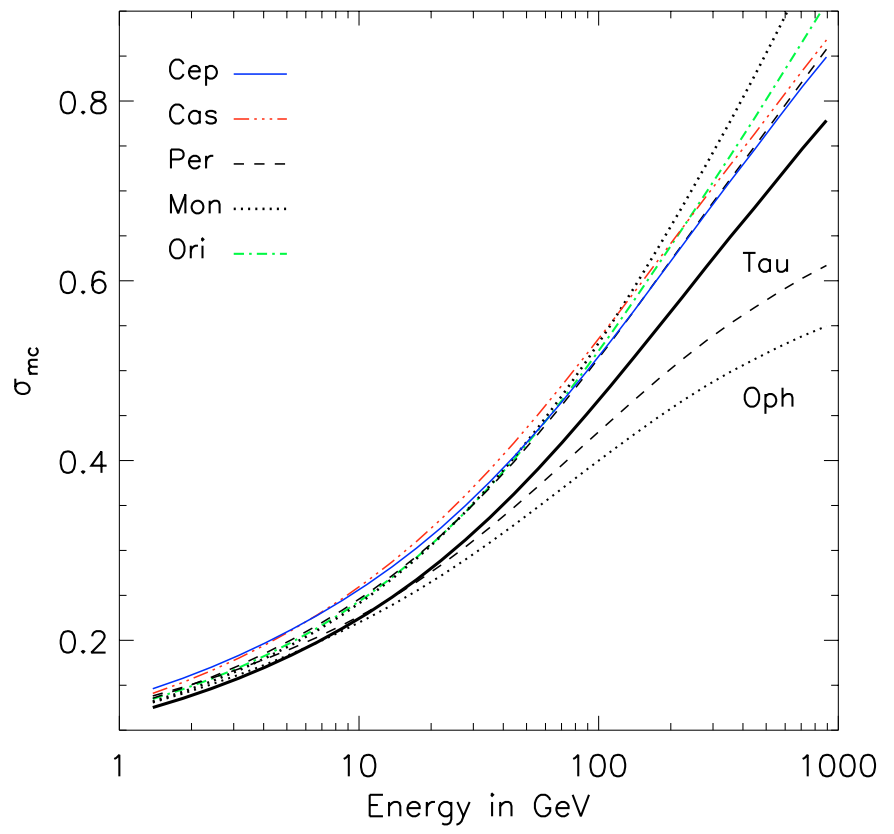

Fig. 6. The amplitude of differences, $\sigma_{\mathrm{mc}}$, in the electron spectra at the molecular clouds and in the solar vicinity. For comparison the thick solid line shows the total dispersion in the local electron spectrum, $\sigma_{\text {abs. }}$. For Taurus and Ophiuchus $\sigma_{\mathrm{mc}}$ is smaller than $\sigma_{\text {abs }}$, indicating that the spectra at the two clouds vary in coordination with that in the solar vicinity.

EGRET data it is possible to derive the local diffuse $\gamma$-ray emissivity spectra for these cloud complexes and to compare them with the line-of-sight integrated spectra in the Galactic plane. The question is: are the diffuse $\gamma$-ray emissivity spectra for the molecular cloud complexes in question compatible with a spatially variable cosmic-ray electron spectrum under the constraints of the Galactic supernova rate and Gould's Belt and the hard inverse Compton model for the $\mathrm{GeV}$ excess?

The limited spatial resolution and count statistics of EGRET data do not permit a detailed analysis of the $\gamma$-ray spectra of small scale structure in the interstellar medium. Published studies of the diffuse $\gamma$-ray emission from isolated molecular clouds are usually based on fitting a model to the $\gamma$-ray data, which consists of a template for the distribution of atomic hydrogen, another template for molecular hydrogen based on the distribution of the CO line intensity, an isotropic component, and possible point sources. The $\gamma$-ray spectra thus derived then are intensity-weighted averages of the actual spectra in different parts of the cloud complexes.

Contributions from inverse Compton scattering and cosmic-ray interactions with ionized hydrogen are often expected to be smooth and hence subsumed with the isotropic emission. At least for the inverse Compton component in lineof-sights near molecular clouds this may be a misperception, for the up-scattering of (structured) far-IR emission near dense molecular clouds may be more important than interactions with the isotropic microwave background, depending on the electron spectrum and on the far-IR energy density. The related $\gamma$-ray intensity would then correlate at some level with the gas column 
density. The infrared radiation field of a molecular cloud falls off approximately $\propto 1 / r^{2}$ with distance from the cloud, $r$. The enhanced $\gamma$-radiation would therefore be observed as a halo around the cloud.

If, on the other hand, the cloud coincides with a strong source of cosmic rays, the correlation length, $l_{\mathrm{c}} \simeq 200 \mathrm{pc}$, of the high-energy electrons indicates that the expected structure in the $\gamma$-ray intensity distribution would be much larger than that of the molecular gas itself.

Thus a possible signature of enhanced inverse Compton emission in the $\gamma$-ray intensity map would have an angular distribution that is different from that of the $\mathrm{CO}$ and $\mathrm{HI}$ line intensity. If one uses only templates for point sources, isotropic emission, the diffuse $\mathrm{HI}$, and the highly structured $\mathrm{CO}$, the fitting routine would have no choice but to associate the IC intensity with whatever template fits best, in many cases presumably the H I distribution.

Studies of the high-energy diffuse $\gamma$-ray emission observed toward isolated, molecular clouds with EGRET are therefore limited in that it is not possible to unambiguously attribute a spectral feature like a $\mathrm{GeV}$ excess to inverse Compton emission. What we can say, though, is that if there is no GeV excess in the data, there is also none in the inverse Compton spectra.

If the $\mathrm{GeV}$ excess was caused by cosmic-ray nucleons, it ought to be visible in the spectrum associated with the $\mathrm{CO}$ template, for it is unlikely that the high-energy cosmic rays would be excluded from the inner cores of the molecular clouds.

The $\gamma$-ray spectra observed toward the nearby clouds Ophiuchus (Hunter et al. 1994) and Taurus (Digel \& Grenier 2001) do not show any evidence for a GeV excess. These complexes are located less than a correlation length, $l_{\mathrm{c}}$, away, so that inverse Compton scattering could not produce excess $\mathrm{GeV}$ emission, in line with the observational result.

The $\gamma$-ray spectra observed toward the more distant Cepheus (Digel et al. 1996), Orion (Digel et al. 1999), and Monoceros clouds (Digel et al. 2001) show evidence for a GeV excess of similar amplitude as that observed toward the inner Galaxy. The excess emission is significant only in the spectra of the Hi template. In the cases of Cepheus and Monoceros the uncertainties in the $\gamma$-ray spectrum associated with the $\mathrm{CO}$ template are too large to permit meaningful conclusions, but for Orion there is simply no evidence of excess emission in the $\mathrm{GeV}$-scale $\gamma$-ray spectrum of the CO template. The spectrum observed toward Perseus (Digel \& Grenier 2001) is in good agreement with that expected for the locally observed cosmicray spectra, and no evidence for excess $\mathrm{GeV}$ emission can be found.

Thus, a GeV excess is observed only toward clouds, which are significantly more distant than one electron correlation length, $l_{c}$. If observed, it is generally associated with the moderately structured $\mathrm{HI}$ template and not significant for the highly-structured $\mathrm{CO}$, indicating it is probably not caused by cosmic ray nucleons. The $\gamma$-ray spectra observed toward the isolated, nearby molecular cloud complexes are therefore consistent with the hard inverse Compton model for the $\mathrm{GeV}$ excess.

\section{Summary}

In this paper we have investigated the effect of Gould's Belt as a local system of enhanced star-forming and hence supernova activity on the local cosmic-ray electron spectrum under the assumption that the electrons are solely produced in SNR. We have assumed that enhanced SN activity commences without delay in the regions which are over-run by the expanding front of Gould's Belt. Hence the Belt is treated as a thick disk as opposed to a shell. In reality this may not be true, in which case the effect of Gould's Belt would be less than calculated here. We have also assumed that the $\mathrm{SN}$ rate per area is constant within the Belt disc.

The time-averaged local electron spectra would be slightly harder than in the case of a homogeneous Galactic disc distribution of SNR. Consequently, the electron source spectra in SNR may be slightly softer than previously thought with a change in spectral index $\Delta s \simeq 0.07$. At the same time the variability induced by the discrete nature of SNR in space and time would be essentially the same as that in the case of homogeneously distributed SNR. The probability of finding the locally observed electron spectrum in the range of possible spectra is thus somewhat reduced, because the calculated spectra are on average slightly harder. Hard electron source spectra and the correspondingly hard inverse Compton $\gamma$-ray spectra have been proposed as a possible explanation of the excess of Galactic GeV $\gamma$-rays observed with EGRET (Pohl \& Esposito 1998). Our results do not rule out this hypothesis, but reduce the available parameter space for such models.

We have also calculated the correlation of the local electron flux with that near seven local molecular cloud complexes, namely Orion, Monoceros, Taurus, Perseus, Cepheus, Cassiopeia, and Ophiuchus. At the nearby clouds Taurus and Ophiuchus ( $D \simeq 140 \mathrm{pc}$ and $D \simeq 125 \mathrm{pc}$, resp.) we find the electron spectra are correlated with that in the solar vicinity, whereas at the more distant clouds $(D \gtrsim 300 \mathrm{pc}$ ) we see no evidence of a correlation. The correlation length for the variations in the electron spectrum must therefore be $l_{\mathrm{c}} \approx 200 \mathrm{pc}$ at energies around $100 \mathrm{GeV}$ and higher. This would permit hard inverse Compton $\gamma$-ray spectra to be associated with some of these cloud complexes. Published studies of the $\gamma$-ray emissivity spectra towards the regions of sky in question indicate that a $\mathrm{GeV}$-excess is evident in Monoceros, Cepheus, and Orion, but only in the spectrum associated with atomic hydrogen. There is no evidence for a $\mathrm{GeV}$-excess in the $\gamma$-ray emission observed toward the nearby clouds Taurus and Ophiuchus, as well as toward Perseus. Our findings are in good agreement with these observational results, thus indicating that the hard inverse Compton model of the GeV-excess is viable.

The conclusions presented here rely on the assumption that cosmic-ray electrons are solely produced in SNR, as a consequence of which the local electron spectrum above $50 \mathrm{GeV}$ may show deviations from power-law behaviour such as bumps and dents. New electron measurements in the high-energy range are urgently required to possibly detect such spectral structures and thereby confirm an electron origin in discrete sources. 
Acknowledgements. This work was supported by the bilateral PROCOPE program. Partial support for MP by the Bundesministerium für Bildung und Forschung through DLR, grant 50 OR 0006, is acknowledged.

\section{References}

Aharonian, F., Akhperjanian, A. G., Barrio, J. A., et al. 2001, A\&A, 370,112

Allen, G. E., Keohane, J. W., Gotthelf, E. V., et al. 1997, ApJ, 487, L97

Barwick, S. W., Beatty, J. J., Bower, C. R., et al. 1998, ApJ, 498, 779

Berezhko, E. G., Pühlhofer, G., \& Völk, H. J. 2003, A\&A, 400, 971

Boezio, M., Carlosn P., Francke, T., et al. 2000, ApJ, 532, 653

Borkowski, K. J., Rho, J., Reynolds, S. P., \& Dyer, K. K. 2001, ApJ, 550,334

Cappelaro, E., Turatto, M., Tsvetkov, D. Yu., et al. 1997, A\&A, 322, 431

Comeròn, F. 1999, A\&A, 351, 506

Comeròn, F., \& Torra, J. 1992, A\&A, 261, 94

Cowsik, R., \& Lee, M.A. 1979, ApJ, 228, 297

de Geus, E. J., Bronfman, L., \& Thaddeus, P. 1990, A\&A, 231, 137

de Zeeuw, P. T., Hoogerwerf, R., de Bruijne, J. H. J., Brown, A. G. A., \& Blaauw, A. 1999, AJ, 117, 354

Digel, S. W., Grenier, I. A. 2001, in Gamma-ray Astrophysics, Proc. of the Gamma 2001 Symp., ed. N. Gehrels, C. Shrader, \& S. Ritz, AIP Conf. Proc., 587, 538

Digel, S. W., Grenier, I. A., Hunter, S. D., Dame, T. M., \& Thaddeus, P. 2001, ApJ, 555, 12

Digel, S. W., Aprile, E., Hunter, S. D., Mukherjee, R., \& Xu, F. 1999 , ApJ, 520, 196

Digel, S. W., Grenier, I. A., Heithausen, A., Hunter, S. D., \& Thaddeus, P. 1996, ApJ, 463, 609

Ferrando, P., Raviart, A., Haasbroek, L. J., et al. 1996, A\&A, 316, 528

Freudenreich, H. T. 1998, ApJ, 492, 495

Ginzburg, V. L., \& Syrovatskii, S. I. 1964, The origin of cosmic-rays (Pergamon Press)

Gleeson, L. J., \& Axford, W. I. 1968, ApJ, 154, 1011

Golden, R. L., Grimani, C., Kimbell, B. L., et al. 1994, ApJ, 436, 769

Golden, R. L., Mauger, B. G., Badhwar, G. D., et al. 1984, ApJ, 287, 622
Green, D. A. 2001, in High Energy Gamma-Ray Astronomy, ed. F. A., Aharonian, \& H. J., Völk, AIP Conf. Proc., 558, 59

Grenier, I. 2000, A\&A, 364, L93

Grenier, I. \& Perrot, C. 2001, in Gamma-ray Astrophysics, ed. N. Gehrels, C. Shrader, \& S. Ritz, AIP Conf. Proc., 587, 649

Grenier, I. A., Lebrun, F., Arnaud, M., Dame, T. M., Thaddeus, P. 1989, ApJ, 347, 231

Grimani, C., Stephens, S. A., Cafagna, F. S., et al. 2002, A\&A, 392, 287

Hammersley, P. L., Garzón, F., Mahoney, T., \& Calbet, X. 1995, MNRAS, 273, 206

Hunter, S. D., Bertsch, D. L., Catelli, J. R., et al. 1997, ApJ, 481, 205

Hunter, S. D., Digel, S. W., de Geus, E. J., \& Kanbach, G. 1994, ApJ, 436, 216

Jones, F. C., Lukasiak, A., Ptuskin, V., \& Webber, W. 2001, ApJ, 547, 264

Keohane, J. W., Petre, R., Gotthelf, E. V., et al. 1997, ApJ, 484, 350

Koyama, K., Petre, R., Gotthelf, E. V., et al. 1995, Nature, 378, 255

Koyama, K., Kinugasa, K., Matsuzaki, K., et al. 1997, PASJ, 49, L7

Maddalena, R. J., Morris, M., Moscowitz, J., \& Thaddeus, P. 1986, ApJ, 303, 375

Maurin, D., Donato, F., Taillet, R., \& Salati, P. 2001, ApJ, 555, 585

Moreno, E., Alfaro, E. J., \& Franco, J. 1999, ApJ, 522, 276

Muraishi, H., Tanimori, T., Yanagita, S., et al. 2000, A\&A, 354, L57

Olano, C. A. 1982, A\&A, 112, 195

Perrot, C. A., \& Grenier, I. A. 2003, A\&A, 404, 525

Pohl, M., \& Esposito, J. A. 1998, ApJ, 507, 327

Pöppel, W. 1997, Fund. Cosm. Phys., 18, 1

Reimer, O., \& Pohl, M. 2002, A\&A, 390, L43

Skibo, J. G. 1993, Ph.D. Thesis, University of Maryland

Slane, P., Gaensler, B. M., Dame, T. M., et al. 1999, ApJ, 525, 357

Strong, A. W., \& Moskalenko, I. V. 1998, ApJ, 509, 212

Strong, A. W., Moskalenko, I. V., \& Reimer, O. 2000, ApJ, 537, 763

Strong, A. W. \& Moskalenko, I. V. 2001, Proc. 27th ICRC, OG 1.3, ed. M. Simon, E. Lorenz, \& M. Pohl, Copernicus Gesellschaft, 1964

Taira, T., Nishimura, J., Fujii, M., et al. 1993, Proc. 23rd ICRC, Calgary, vol. 2, 128

Tang, K.K. 1984, ApJ, 278, 881

Tanimori, T., Hayami, Y., Kamel, S., et al. 1998, ApJ, 497, L25

Torii, S., Tamura, T., Tateyama, N., et al. 2001, ApJ, 559, 973

Ungerechts, H., \& Thaddeus, P. 1987, ApJS, 63, 645

Webber, W.R., Lee, M.A., \& Gupta, M. 1992, ApJ, 390, 96 